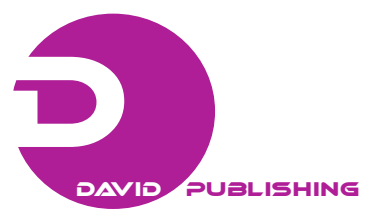

\title{
Use of ANSYS for Thermal Analysis in Mass Concrete
}

\author{
Nailde de Amorim Coelho, Lineu José Pedroso, João Henrique da Silva Rêgo and Antônio Alberto Nepomuceno \\ Department of Civil and Environment Engineering, University of Brasilia, Brasilia 70.919-970, Brazil
}

\begin{abstract}
With the increasing development of Brazil in recent years, major engineering construction works have been designed and built, particularly those involving large volumes of mass concrete, such as in the case of dams. Mass concrete, due to its large size and volume, presents a considerable temperature rise caused by cement grain hydration. This temperature rise can be sufficient to cause concrete crack and/or cracking, which may lead to serious problems. In this paper, we sought to study heat generation and temperature field in mass concrete through ANSYS software, which uses finite element method to analyze the problem. This program allows temperatures to be checked for different concrete ages. With that, it is possible to evaluate the temperatures obtained and the factors influencing the results in a short period of time at a low cost. With the help of the software, it is possible to check the temperatures for different concrete properties by analyzing them on different concreting days. Therefore, it was possible to establish that the properties of the concrete directly influence the temperature evolution phenomenon.
\end{abstract}

Key words: Temperature, mass concrete, isotherms, numerical analysis.

\section{Introduction}

Mass concrete is any volume of concrete with dimensions large enough to require measures to be taken to overcome heat generation from cement hydration, by reducing the volume change in order to minimize cracking [1].

Many construction works use concrete mass in their making. Such structures typically have large sizes and large volumes of concrete and become a concern due to the likelihood of the development of pathologies such as cracking and cracks. When cement grains come into contact with water, they hydrate and release heat, which is known as heat of hydration.

Heat generation inside a concrete block generates a temperature gradient in relation to the external edges which, if the concrete cannot move freely, induces the appearance of a tensile stress which, if it exceeds the tensile strength of the concrete, will cause cracking and possibly damages and tightness to the structure [2]. Many studies can be found in literature about the phenomenon related to the thermal effects on mass concrete [3-7].

Corresponding author: Nailde de Amorim Coelho, M.Sc., research fields: structures and civil construction. E-mail: naildea@yahoo.com.br.
Some resources, such as the use of software, can predict and assess the internal temperature of mass concrete. This process allows the identification of the highest temperature achieved, heat evolution time and critical temperature spots on the structure. With this data, it is possible to find solutions more suited to the problem.

An example of software that can be used in such problems is ANSYS, a powerful tool that uses finite element method for solving many engineering problems of a structural, dynamic and thermal nature, among others. It provides a fast and economical analysis when compared to experimental tests.

Thus, in this paper, ANSYS will be used as the operational tool for visualizing thermal effects and temperature variation inside mass concrete structures. Thermal variation arising from changes to the parameters in the equation which govern the problem of heat inside the structure will be studied [8-11].

\section{Thermal Properties of the Concrete}

The study of specific mass, specific heat, thermal conductivity and diffusivity is important for the development of temperature gradients, thermal 
deformations and concrete early-age cracking. Below are brief definitions of each:

- Specific mass $(\rho)$-The ratio between the mass of a body and the volume that this body occupies. It is influenced by water and fines content, aggregates used and the voids index. The smaller the amount of water and paste content in the mixture the greater will be the specific mass;

- Thermal conductivity (k)-The material's capability of conducting heat, which is defined as the ratio between heat flow and temperature gradient. Thermal conductivity depends on the composition of concrete and increased humidity also makes its value increase [12];

- Specific heat $(c)$-A physical parameter which expresses the ability of a material to store heat. The specific heat is the amount of heat required to increase the temperature of a mass unit in one degree. Typical specific heat values for normal concrete are between 0.84 and $1.26 \mathrm{~kJ} /(\mathrm{kg} \cdot \mathrm{K})$ [13];

- Thermal diffusivity $(\delta)$ - It represents the speed at which the temperature varies inside a mass and is therefore an index of the ease with which the concrete can undergo temperature variations. According to the literature [14], thermal diffusivity is the property that expresses heat diffusion capacity in all directions and indicates the ease with which the concrete tolerates temperature variations. It is calculated according to Eq. (1).

$$
\delta=\frac{k}{c \rho}
$$

where,

$k$ = thermal conductivity;

$c=$ specific heat;

$\rho=$ specific mass of the concrete.

In mass concrete structures, the effect of cement hydration produces an exothermic reaction with significant temperature rise of this material. Besides heat of hydration, there are other thermal phenomena that act directly on these constructions: solar radiation, conduction and convection. A mass concrete structure generates heat in the first few moments of its existence, conducts heat through its mass; receives, emits and reflects heat through its faces and, over time, reaches its thermal equilibrium.

\section{Theoretical Foundations}

One of the main objectives of analyzing thermal effects is to determine the temperature field in a medium resulting from the conditions imposed on its borders. That is, the intention is to know the distribution of temperatures, which represents how the temperature varies with the position in the medium. Once that distribution is known, heat flow by conduction on any point in the medium or on the surface can be determined by Fourier's law [15].

Consider a homogeneous medium, and a temperature distribution $T(x, y, z)$ represented in Cartesian coordinates. The general heat transfer equation is evaluated by the heat flow that passes through that body, as is shown in Fig. 1 [8-11].

$\mathrm{Be} c$ the specific heat of the material, $k$ the thermal conductivity; $\rho$ its specific mass, $(\Delta x \Delta y \Delta z)$ the volume element and $\left(\frac{d T}{d x}\right)$ the thermal gradient in the $x$ direction. The volume element will experience, in the time unit, a temperature rise as a function of time, $\left(\frac{d T}{d t}\right)$ and the Fourier's equation shown in Eqs. (2) and (3) is obtained:

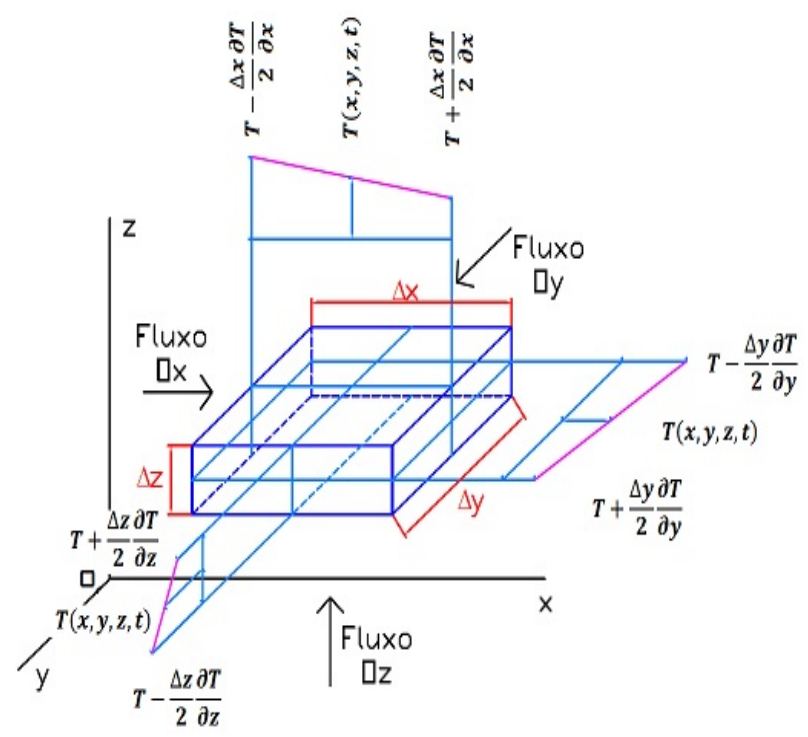

Fig. 1 Body subjected to a heat flow. 


$$
\begin{gathered}
k \Delta x \Delta y \Delta z\left(\frac{\partial^{2} T}{\partial x^{2}}+\frac{\partial^{2} T}{\partial y^{2}}+\frac{\partial^{2} T}{\partial z^{2}}\right)=c \rho \Delta x \Delta y \Delta z \frac{\partial T}{\partial t} \\
k \nabla^{2} T=c \rho \frac{\partial T}{\partial t}
\end{gathered}
$$

where: $\nabla^{2} T=\frac{\partial^{2} T}{\partial x^{2}}+\frac{\partial^{2} T}{\partial y^{2}}+\frac{\partial^{2} T}{\partial z^{2}}$.

If the body develop heat, as is the case with concrete, there is the influence of another term in Fourier's equation which is a representation of the amount of heat developed, given by $\dot{q}$. Therefore, Eq. (4) is obtained:

$$
k \nabla^{2} T+\dot{q}=c \rho \frac{\partial T}{\partial t}
$$

Thus, the general equation of heat conduction, considering Fourier's equation and the inner heat generation (heat of hydration), is given by Eq. (5):

$$
\nabla^{2} T+\frac{\dot{q}}{k}=\frac{c \rho}{k} \frac{\partial T}{\partial t}
$$

Eq. (5) implies that at any point in the medium the net rate of energy transfer by conduction to the interior of a unit volume plus the volumetric rate of thermal energy generation must be equal to the rate of variation of the thermal energy accumulated inside this volume [16].

In order to solve Fourier's equation, described in Eq. (5), it is necessary to consider heat of hydration $\dot{q}$ for the case of the concrete. The most recent study on the Three Gorges dam in China [15], states that the heat developed by cement hydration can be represented by Eq. (6), obtained through experimental data, taking into consideration the adiabatic temperature at different ages of the concrete.

$$
\dot{q}=q_{i}\left(1-e^{-\alpha t^{\beta}}\right)
$$

where,

$\dot{q}=$ heat of hydration;

$\dot{q}, \alpha, \beta,=$ constants obtained experimentally, which are dependent on the cement composition;

$q_{i}=$ is the initial heat of the concrete.

\section{Results Obtained with the Aid of ANSYS Software}

Analysis of characteristic parameters present in the general equation, for the analysis of the parameters used in Fourier's equation for mass concrete structures, a $5 \mathrm{~m} \times 5 \mathrm{~m}$ concrete block with $0.10 \mathrm{~m}, 2,601$ nodes mesh and 2,500 elements was studied in ANSYS. The element used was PLANE55 from the ANSYS library, which has thermal conduction capacity in two dimensions, 2D, sufficiently tested and externally evaluated. The element has four nodes and a single degree of freedom at each node, the temperature [13].

The temperature isotherms were analyzed after two, four and six days of concreting. For all the cases, the variables corresponding to each property are listed as follows:

$k=$ Thermal conductivity;

$\rho=$ Density;

$c=$ Specific heat;

$h=$ Convection coefficient;

$\Delta(\%)=$ Temperature difference in percentage.

It is noteworthy that for the analyzed parameters, the minimum and maximum values allowed for the concrete were assumed. And these were rounded to integer values, to the nearest immediately lower integer number in the case of minimal values, and to the immediately higher integer number in the case of maximum values.

Another important remark is that for this study the parameters were considered as separately varying, i.e., without depending on other parameters. However, it is known, for instance, that if the trace of the concrete is changed, there normally is a change to all parameters and not only to just one as is being adopted.

In order to clarify the dependencies of these parameters, Table 1 shows them.

For the analysis, $20{ }^{\circ} \mathrm{C}$ ambient temperature, $25{ }^{\circ} \mathrm{C}$ temperature in the lower face of the block, and $45{ }^{\circ} \mathrm{C}$ concrete placement temperature were assumed. The results of the characteristic parameters are shown in Figs. 2-7 and in Tables 2-4. It is worth mentioning that the maximum temperature node is indicated as the black dot in Fig. 2.

\subsection{Thermal Conductivity}

Table 2, and Figs. 2 and 3 summarize the main results obtained for the two values (minimum and maximum) 
Table 1 Definition of parameters.

\begin{tabular}{|c|c|c|}
\hline Parameter & Definition & Dependence \\
\hline Thermal conductivity $k$ & Material's ability to conduct heat & $\begin{array}{l}\text { - Concrete composition; } \\
\text { - Moisture content; } \\
\text { - Mineralogical characteristics of the aggregate; } \\
\text { - Concrete density and temperature; } \\
\text { - It is not influenced by the type of cement used. }\end{array}$ \\
\hline Specific mass or density & $\begin{array}{l}\text { It is the ratio between body mass and the } \\
\text { volume that this body occupies }\end{array}$ & $\begin{array}{l}\text { - Water and fines content; } \\
\text { - Aggregates used; } \\
\text { - Voids index; } \\
\text { - Concrete temperature. }\end{array}$ \\
\hline Specific heat $c$ & $\begin{array}{l}\text { Corresponds to the amount of heat required } \\
\text { to increase the temperature of one unit of } \\
\text { mass in one degree }\end{array}$ & $\begin{array}{l}\text { - Temperature; } \\
\text { - Specific mass of the concrete; } \\
\text { - Moisture content; } \\
\text { - Characteristic maximum size of coarse aggregate. }\end{array}$ \\
\hline
\end{tabular}

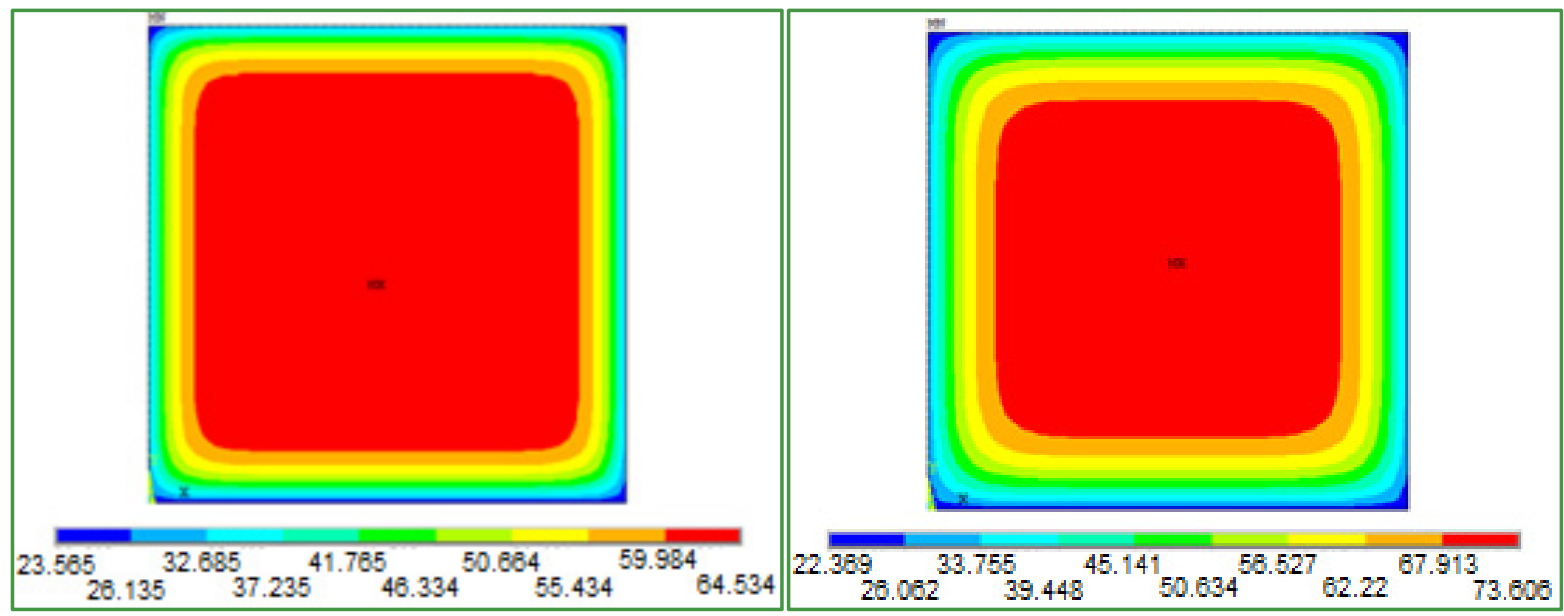

(a)

(b)

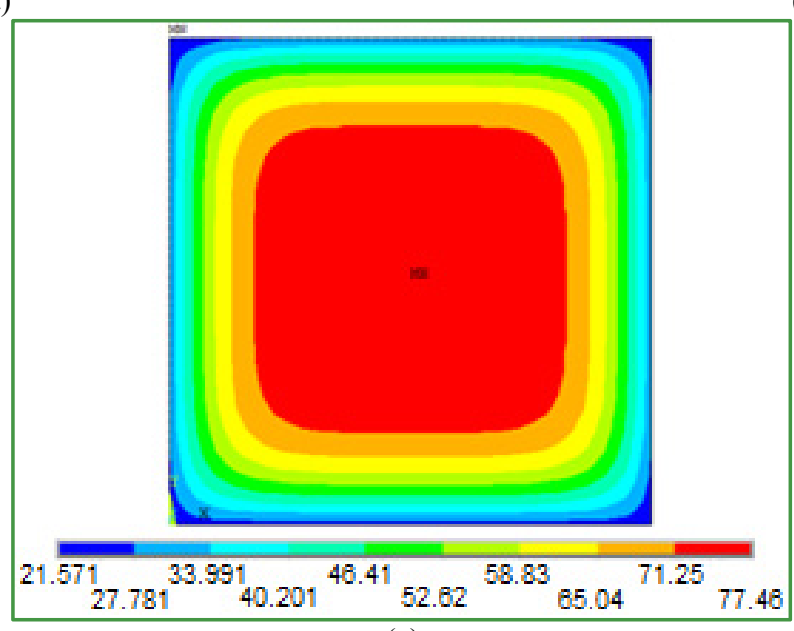

(c)

Fig. 2 Temperature isotherms for 2 (a), 4 (b) and 6 (c) concreting days, with $k=1 \mathrm{~W} / \mathrm{m} \cdot{ }^{\circ} \mathrm{C}$.

of thermal conductivity of the concrete.

It is noted that the lower the thermal conductivity of the concrete, the higher the maximum temperature reached. This can be explained by the fact that with a low thermal conductivity, the greater will be the difficulty of dissipating heat internally generated in this body. It is noted also that the "time" parameter directly influences these isotherms. Eventually, it is observed 
Table 2 Results for different values of $k$.

\begin{tabular}{llll}
\hline Properties & \multicolumn{3}{c}{ Maximum temperature; Node } \\
\cline { 2 - 4 } & 2 days & 4 days & 6 days \\
\hline$k=1 \mathrm{~W} / \mathrm{m}^{\circ} \mathrm{C}$ & $64.534{ }^{\circ} \mathrm{C}$ & $73.606{ }^{\circ} \mathrm{C}$ & $77.460{ }^{\circ} \mathrm{C}$ \\
$\rho=2,300 \mathrm{~kg} / \mathrm{m}^{3}$ & Node $=1,401$ & Node $=1,401$ & Node $=1,401$ \\
$c=1,000 \mathrm{~J} / \mathrm{g} \cdot{ }^{\circ} \mathrm{C}$ & & & \\
$h=10 \mathrm{~W} / \mathrm{m}^{2} \cdot{ }^{\circ} \mathrm{C}$ & & $71.874{ }^{\circ} \mathrm{C}$ & $71.375{ }^{\circ} \mathrm{C}$ \\
\hline$k=4 \mathrm{~W} / \mathrm{m}^{\circ} \mathrm{C}$ & & Node $=1,402$ & Node $=1,402$ \\
$\rho=2,300 \mathrm{~kg} / \mathrm{m}^{3}$ & Node $=1,402$ & \\
$c=1,000 \mathrm{~J} / \mathrm{g}^{\circ} \mathrm{C}$ & & 2.41 & 8.52 \\
$h=10 \mathrm{~W} / \mathrm{m}^{2} \cdot{ }^{\circ} \mathrm{C}$ & 0.11 & & \\
\hline$\Delta(\%)$ & & &
\end{tabular}

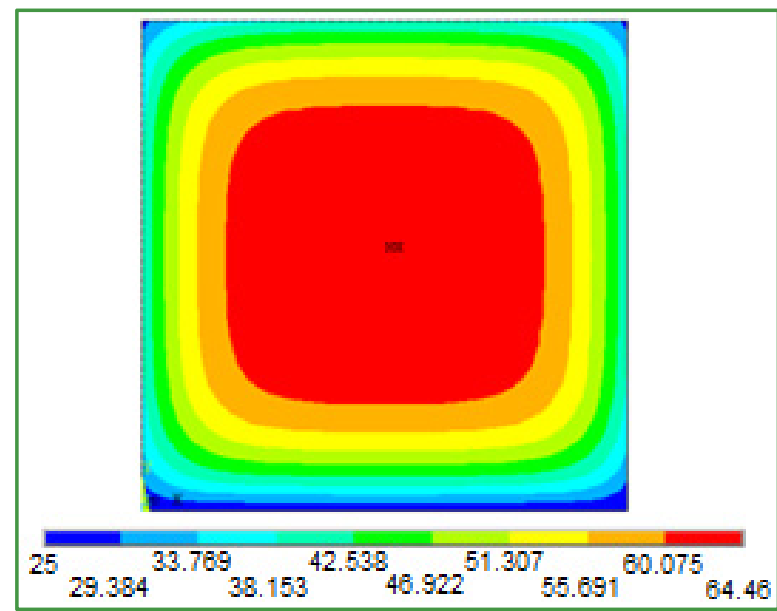

(a)

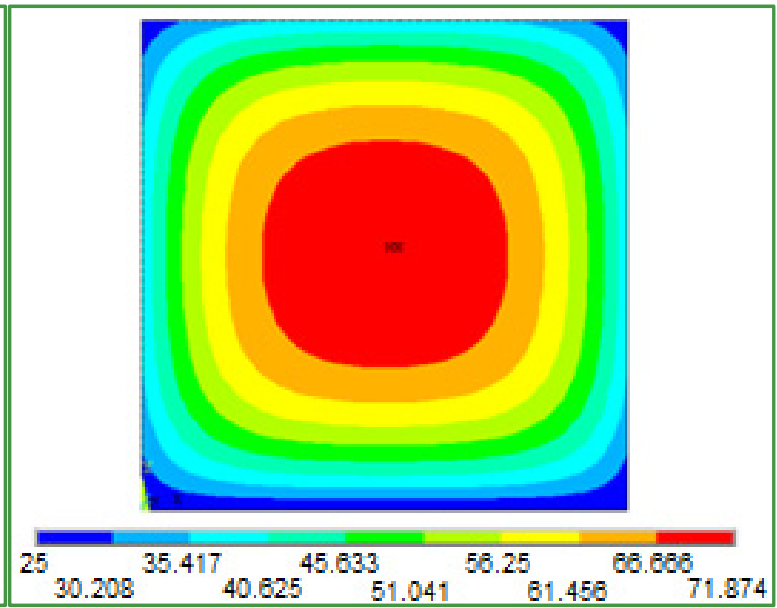

(b)

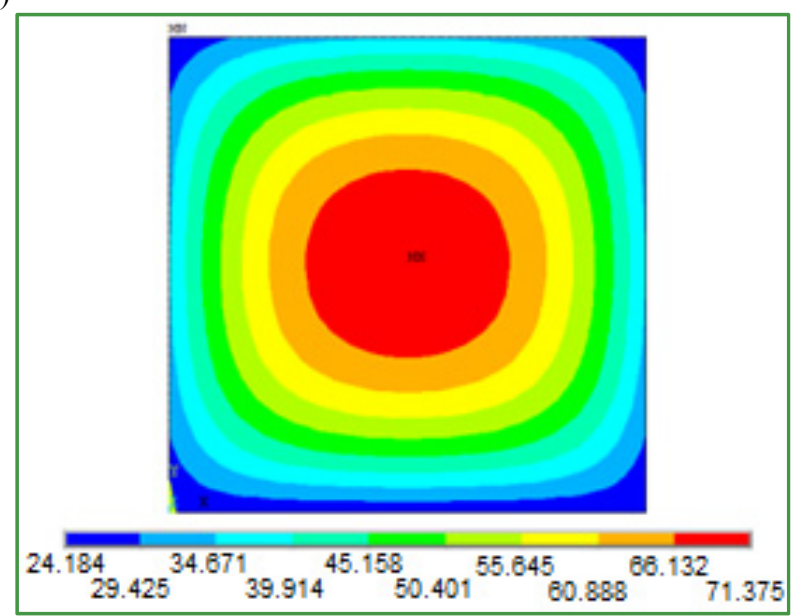

(c)

Fig. 3 Temperature isotherms for 2 (a), 4 (b) and 6 (c) concreting days, with $k=4 \mathrm{~W} / \mathrm{m}^{\cdot}{ }^{\circ} \mathrm{C}$.

that the region with the maximum temperature becomes smaller.

\subsection{Specific Heat}

Table 3 and Figs. 4 and 5 summarize the results obtained for two extreme values of the specific heat of the concrete.

It is known that specific heat corresponds to the amount of heat required to increase the temperature of a mass unit in one degree; and that it reaches a maximum value for saturated concrete. It is observed that for a lower value of specific heat, higher 
temperatures are obtained, i.e., less heat is required in order for a temperature rise to occur. However, for a higher value of specific heat, the maximum temperature isotherms are higher than in the case of lower specific heat.

\subsection{Specific Mass}

Table 4 and Figs. 6 and 7 show the results obtained for two different values (extreme) of the specific mass of the concrete.

With the reduction in specific mass there is an increase in temperature. It is known that specific mass is the ratio between the mass of a body and the volume this body occupies; and that with the reduction of water/cement ratio in the mixture there is an increase in its specific mass. Thus, it can be said that for a lower

Table 3 Results for different values of $c$.

\begin{tabular}{llll}
\hline Properties & \multicolumn{2}{c}{ Maximum temperature; Node } \\
\cline { 2 - 4 } & 2 days & 4 days & 6 days \\
\hline$k=1.79 \mathrm{~W} / \mathrm{m}^{\circ}{ }^{\circ} \mathrm{C}$ & & $80.549{ }^{\circ} \mathrm{C}$ & $84.179{ }^{\circ} \mathrm{C}$ \\
$\rho=2,300 \mathrm{~kg} / \mathrm{m}^{3}$ & $69.416{ }^{\circ} \mathrm{C}$ & Node $=1,401$ & Node $=1,402$ \\
$c=800 \mathrm{~J} / \mathrm{g} \cdot{ }^{\circ} \mathrm{C}$ & Node $=1,401$ & & \\
$h=10 \mathrm{~W} / \mathrm{m}^{2} \cdot{ }^{\circ} \mathrm{C}$ & & & $69.856{ }^{\circ} \mathrm{C}$ \\
\hline$k=1.79 \mathrm{~W} / \mathrm{m}^{\circ} \mathrm{C}$ & & $66.996{ }^{\circ} \mathrm{C}$ & Node $=1,401$ \\
$\rho=2,300 \mathrm{~kg} / \mathrm{m}^{3}$ & $60.026{ }^{\circ} \mathrm{C}$ & Node $=1,401$ & 20.50 \\
$c=1,300 \mathrm{~J} / \mathrm{g} \cdot{ }^{\circ} \mathrm{C}$ & Node $=1,401$ & & \\
$h=10 \mathrm{~W} / \mathrm{m}^{2} \cdot{ }^{\circ} \mathrm{C}$ & & 20.23 & \\
\hline$\Delta(\%)$ & 15.64 & & \\
\hline
\end{tabular}

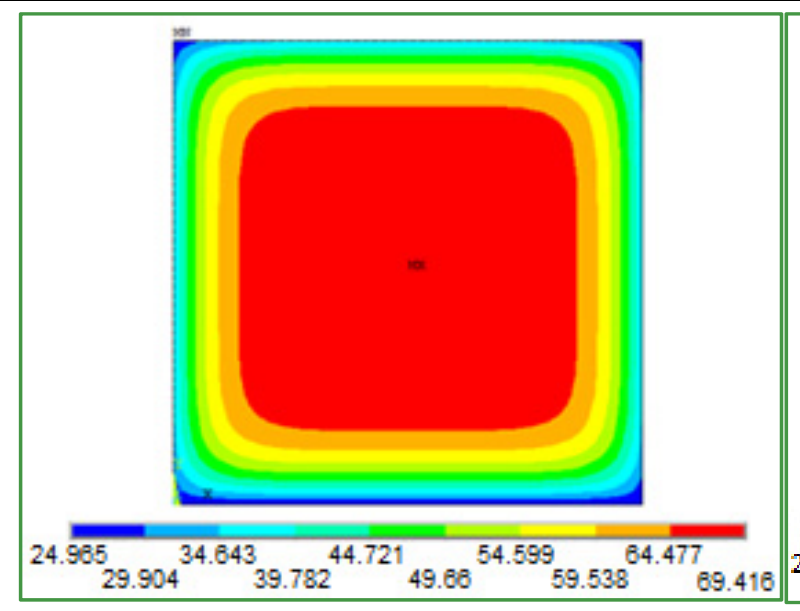

(a)

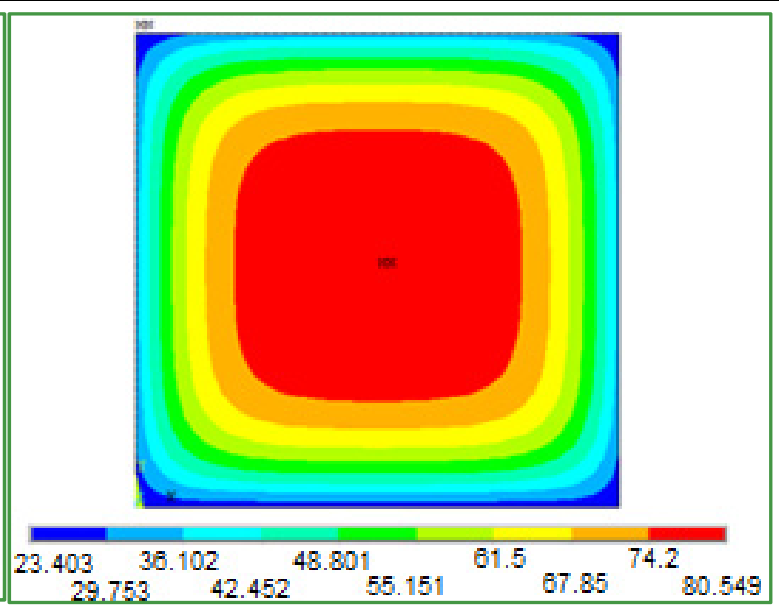

(b)

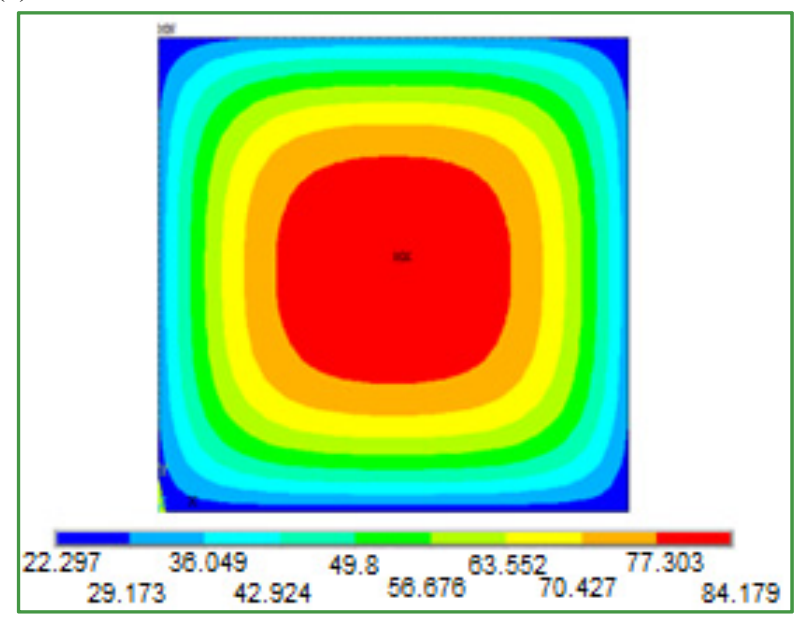

(c)

Fig. 4 Temperature isotherms for 2 (a), 4 (b) and 6 (c) concreting days, with $c=800 \mathrm{~J} / \mathrm{g} .{ }^{\circ} \mathrm{C}$. 


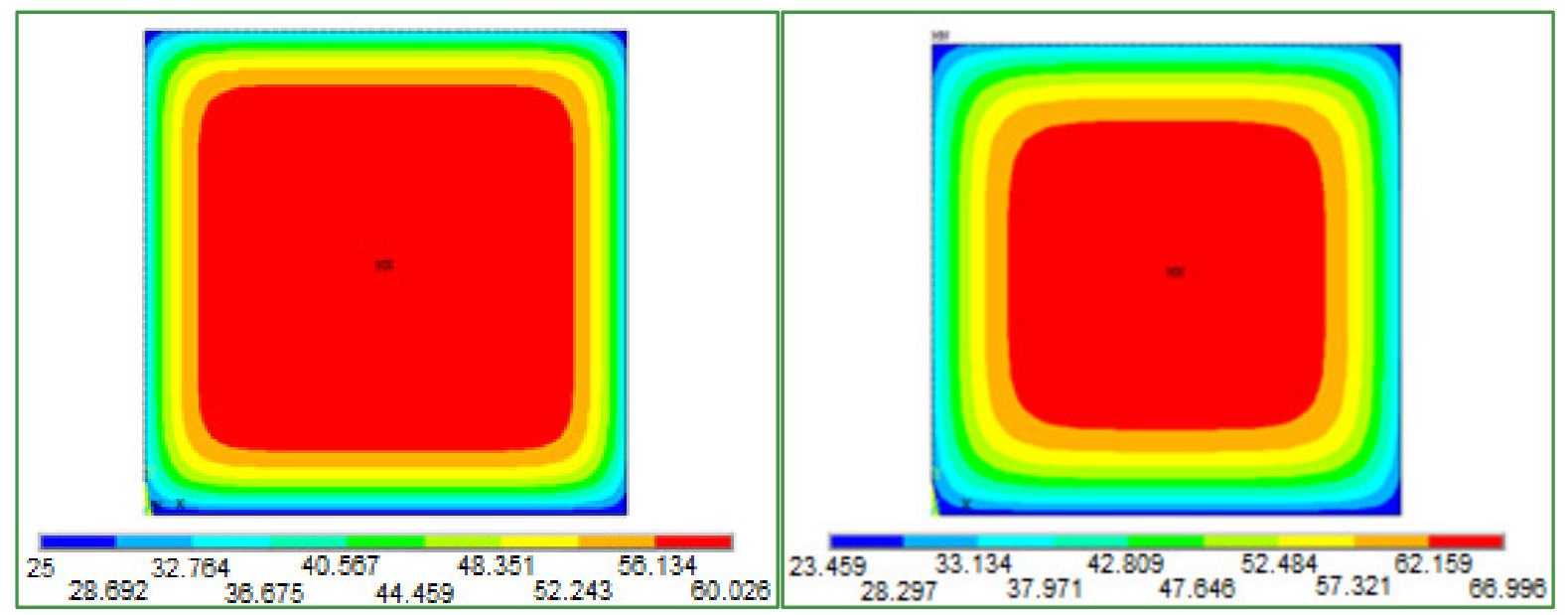

(a)

(b)

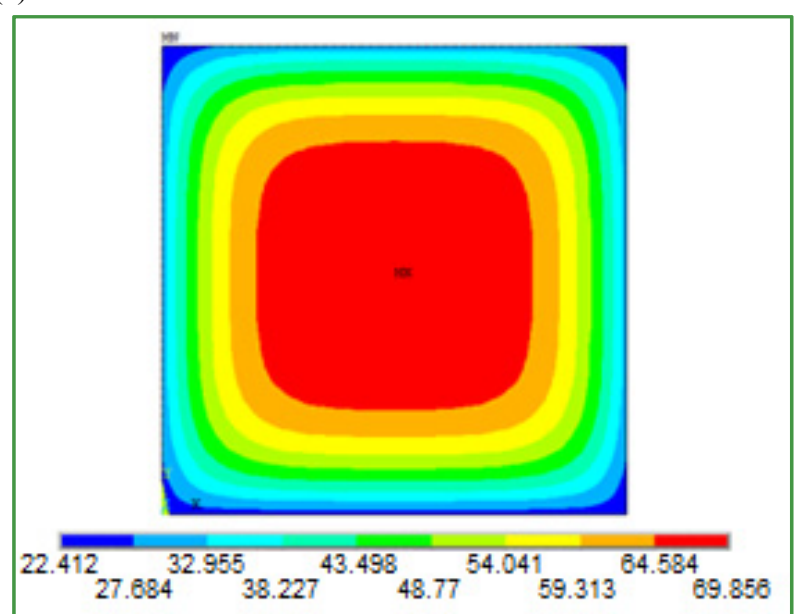

(c)

Fig. 5 Temperature isotherms for 2 (a), 4 (b) and 6 (c) concreting days, with $c=1,300 \mathrm{~J} / \mathrm{g} .{ }^{0} \mathrm{C}$.

Table 4 Results for different values of $\rho$.

\begin{tabular}{llll}
\hline Properties & \multicolumn{2}{c}{ Maximum temperature; Node } \\
\cline { 2 - 4 } & 2 days & 4 days & 6 days \\
\hline$k=1.79 \mathrm{~W} / \mathrm{m}^{\circ}{ }^{\circ} \mathrm{C}$ & $67.463{ }^{\circ} \mathrm{C}$ & $77.767{ }^{\circ} \mathrm{C}$ & $81.358^{\circ} \mathrm{C}$ \\
$\rho=2,000 \mathrm{~kg} / \mathrm{m}^{3}$ & Node $=1,401$ & Node $=1,401$ & Node $=1,401$ \\
$c=1,000 \mathrm{~J} /{ }^{\circ} \mathrm{C}$ & & & \\
$h=10 \mathrm{~W} / \mathrm{m}^{2} \cdot{ }^{\circ} \mathrm{C}$ & & $66.923{ }^{\circ} \mathrm{C}$ & $69.775^{\circ} \mathrm{C}$ \\
\hline$k=1.79 \mathrm{~W} / \mathrm{m}^{\circ}{ }^{\circ} \mathrm{C}$ & $59.976{ }^{\circ} \mathrm{C}$ & Node $=1,401$ & Node $=1,401$ \\
$\rho=3,000 \mathrm{~kg} / \mathrm{m}^{3}$ & Node $=1,401$ & & 16.60 \\
$c=1,000 \mathrm{~J} / \mathrm{g}^{\circ} \mathrm{C}$ & & 16.20 & \\
$h=10 \mathrm{~W} / \mathrm{m}^{2} \cdot{ }^{\circ} \mathrm{C}$ & 12.48 & & \\
\hline$\Delta(\%)$ & &
\end{tabular}

specific mass there is higher insulation, which makes the dissipation of internal heat more difficult.

\section{Conclusions}

One of the factors that have the greatest influence on thermal effects is internal heat generation in the concrete. When cement grains come into contact with water they hydrate and release heat. This heat generation causes thermal stresses that can damage the structure. However, these stresses are more significant when there is a larger temperature gradient, that is, when there is a greater difference between the 
maximum and minimum temperatures in the mass concrete.

Various analyzes have been performed for structures in mass concrete, where thermal effects could be

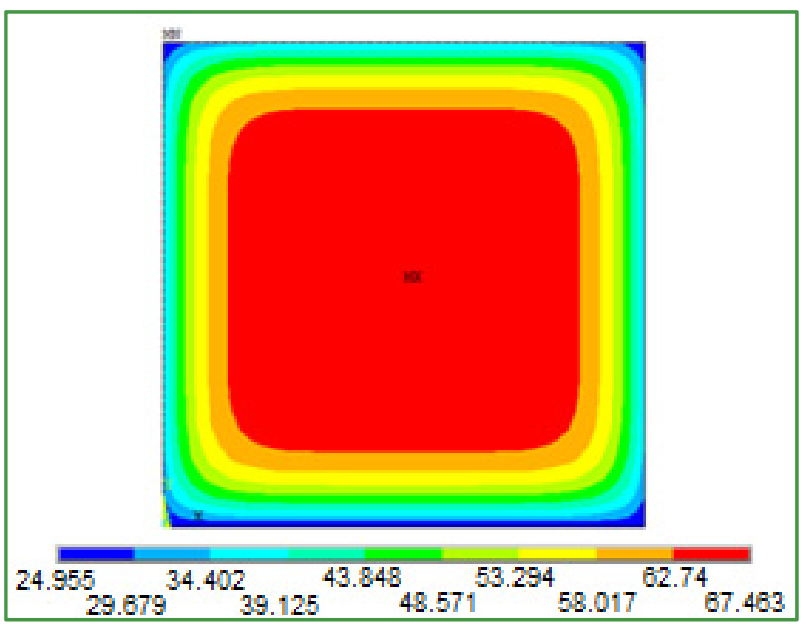

(a)

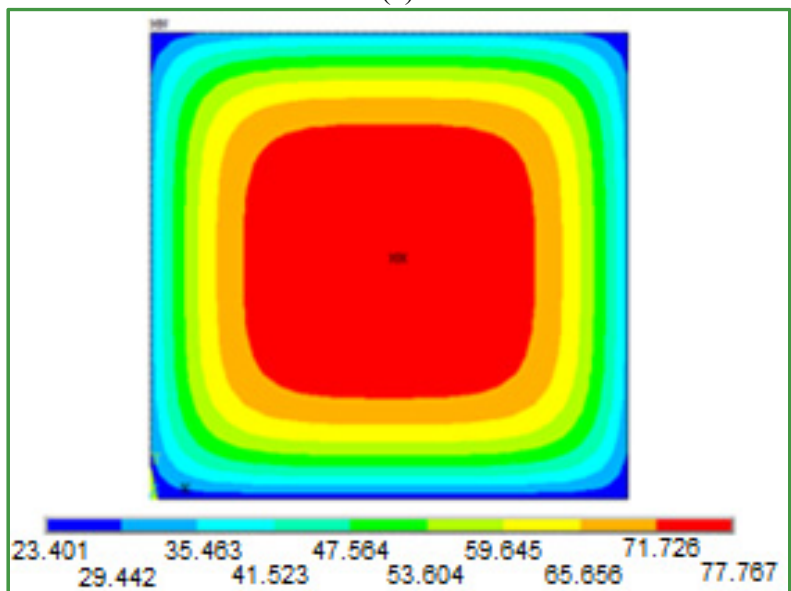

(b)

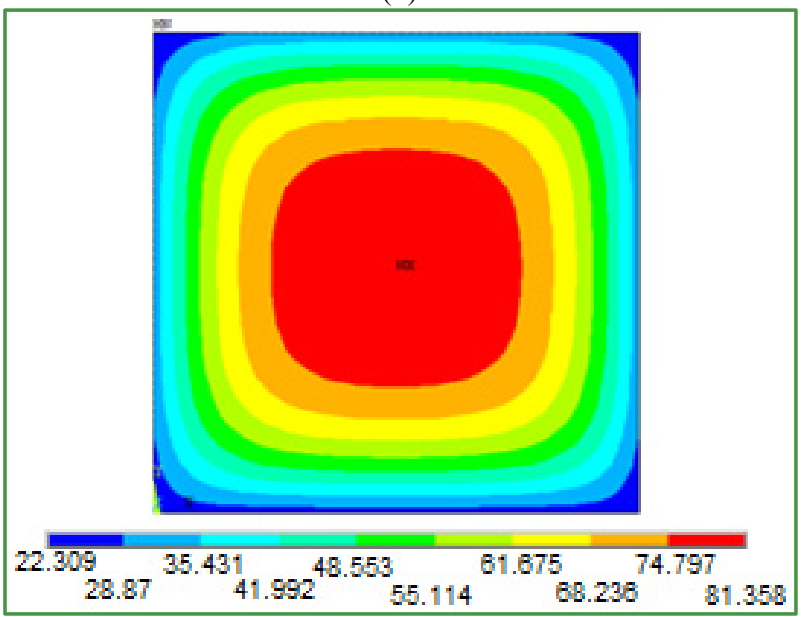

(c)

Fig. 6 Temperature isotherms for 2 (a), 4 (b) and 6 (c) concreting days, with $\rho=2,000 \mathrm{~kg} / \mathrm{m}^{3}$. analyzed through temperature isotherms obtained using ANSYS software. These isotherms indicate the evolution of heat, showing the temperatures within the studied body.

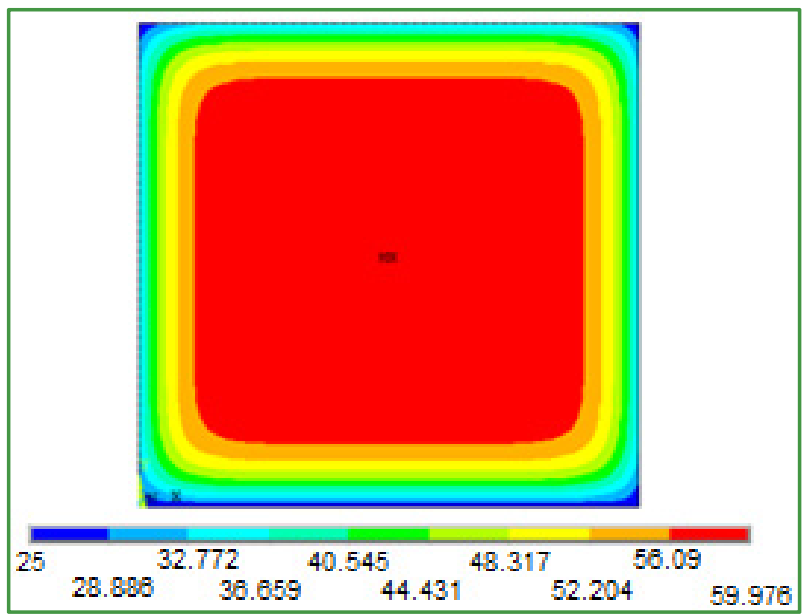

(a)

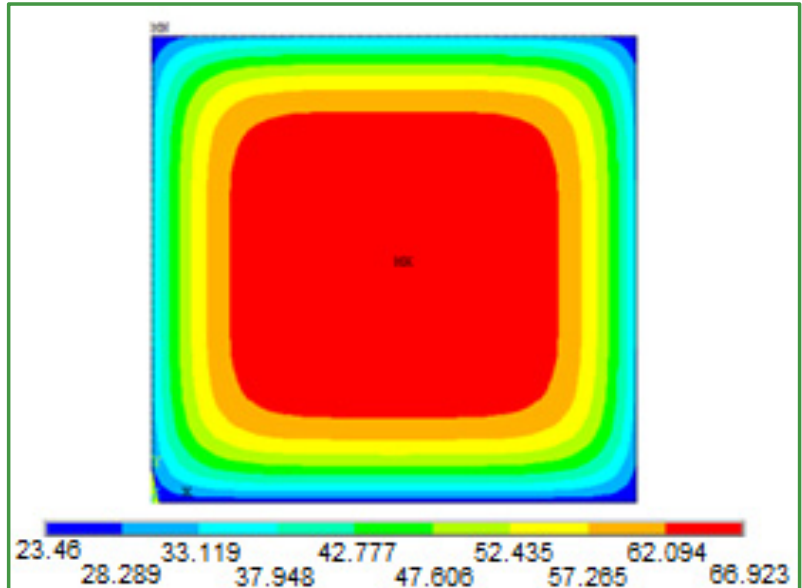

(b)

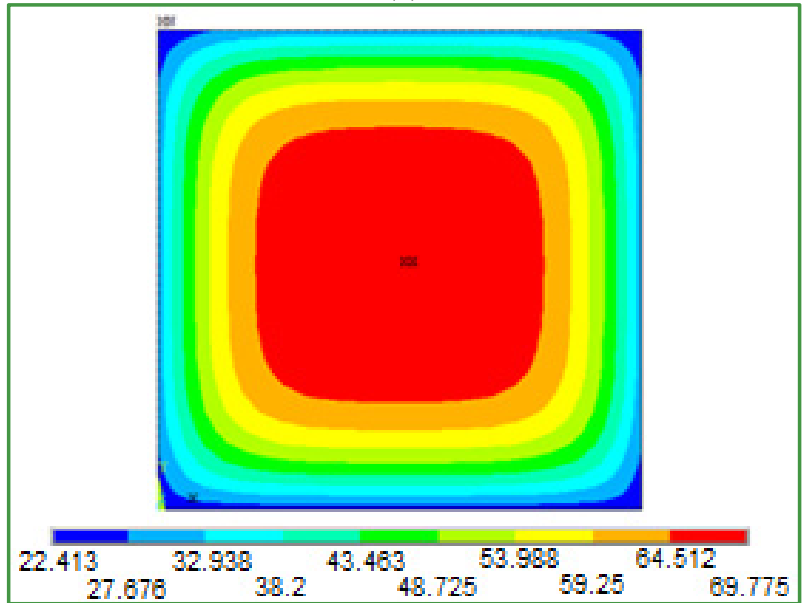

(c)

Fig. 7 Temperature isotherms for 2 (a), 4 (b) and 6 (c) concreting days, with $\rho=3,000 \mathrm{~kg} / \mathrm{m}^{3}$. 
It was noted that a lower thermal conductivity of the concrete implies a higher maximum temperature due to the difficulty of dissipating internally generated heat in the body. With the reduction in specific mass there is an increase in temperature. This happens because they have greater porosity, which hinders the dissipation of internal heat. For a lower specific heat the lower the value, the higher the temperatures, because a smaller amount of heat is required in order for a temperature rise to occur. Thus, it is clear that in order to reduce the internal heat a concrete with higher thermal conductivity, density and specific heat is desirable. However, these properties establish a relationship of dependency that was not considered in this paper.

It was also verified that using a program based on finite element method to solve the problems can be a quick and practical means for a thermal analysis in structures. It enables structures to be analyzed before construction, thus allowing strategies for reducing stress arising from thermal effects.

The mass concrete must have a higher thermal conductivity, density and specific heat, because these features reduce the internal temperature of the concrete and reduce the possibility of cracks due to thermal gradient. Thus, it is necessary to study the thermal parameters and its influence more thoroughly, including experimentally. With this, one can create mass concrete mixtures that correspond to smaller generation of heat of hydration. It is also recommended to make a study of trustworthiness due to uncertainties in the parameters used.

\section{Acknowledgments}

Thanks to the team at PECC-Post-Graduate Program in Structures and Civil Construction of UNB-Universidadede Brasilia.

\section{References}

[1] Cement and Concrete Terminology, ACI 318 Farmington Hills, American Concrete Institute, 2005.

[2] G. Inoue, Preventive Measures to Control Temperature Induces Cracking in Mass, Technical Bulletin \#7, Polytechnic School of the University of São Paulo, São
Paulo, 1986. (in Portuguese)

[3] M.A.D. Azenha, Numerical simulation of the structural behaviour of concrete since its early ages, Dr. Thesis, Engineering Department, University of Porto, Porto, 2009.

[4] P. Choktaweekarn, S. Tangtermsirikul, Effect of aggregate type, casting, thickness, and curing condition on restrained strain of mass concrete, Songklanakarin Journal of Science and Technology 32 (4) (2010) 391-402. (in Portuguese)

[5] E.E. Kavamura, Study of the behavior of two-dimensional thermo-structural concrete dams using the finite element method, M.Sc. Dissertation, Federal University of Paraná, State of Curitiba, 2005. (in Portuguese)

[6] D.A.V. Krüger, Transient thermal analysis of concrete structures performed by layers, M.Sc. Dissertation, Federal University of Paraná, State of Curitiba, 2001. (in Portuguese)

[7] S.B. Santos, A contribution to the study of the thermomechanical behavior of massive concrete structures. linear viscoelastic modeling and applications, M.Sc. Dissertation, Federal University of Espírito Santo, Vitoria, 2004, p. 287. (in Portuguese)

[8] N.A. Coelho, A numerical study of thermal effect in mass concrete, M.Sc. Dissertation, University of Brasília, Brasília, 2012.

[9] N.A. Coelho, L.J. Pedroso, J.H.S. Rêgo, A.A. Nepomuceno, Influence of the thermal properties of the concrete mass analysis of the temperature in large structures, in: 10th World Congress in Computational Mechanics, São Paulo, 2012. (in Portuguese)

[10] N.A. Coelho, L.J. Pedroso, J.H.S. Rêgo, A.A. Nepomuceno, A numerical study of thermal effects caused by heat of hydration of cement in Dams, in: 54th Brazilian Concrete Congress, Maceió, 2012 (in Portuguese)

[11] N.A. Coelho, L.J. Pedroso, J.H.S. Rêgo, A.A. Nepomuceno, Numerical analysis and thermal of mass concrete blocks using a computer code, in: 54th Brazilian Concrete Congress. Maceió, 2012. (in Portuguese)

[12] A.M. Neville, Properties of Concrete, 2nd ed., Pini Publisher, Sao Paulo, 1997, p. 828. (in Portuguese)

[13] E.F. Faria, Exothermy prediction of concrete hydration reaction by thermo-chemical and data modeling, M.Sc. Dissertation, Federal University of Rio de Janeiro, Rio de Janeiro, 2004, p. 145.

[14] NBR 12818: Concrete-Determination of Thermal Diffusivity, ABNT-Brazilian Association of Technical Standards, Rio de Janeiro,1993. (in Portuguese)

[15] F.P. Incropera, D.P. Dewitt, T.L. Bergman, A.S. Lavine, Fundamentals of Heat Transfer and Mass Transfer, 6th ed. LTC, Rio de Janeiro, 2008. (in Portuguese)

[16] J. Liu, L. Qiao, P. Li, C. Dai, Laboratory test and numerical simulation of time-dependent thermo mechanical behavior of the three-gorges dam, Journal of Materials in Civil Engineering 22 (2) (2010) 111-123. (in Portuguese). 